\title{
Deformation Test System of Transmission Tower Based on Binocular Vision Method
}

\author{
Haikun Jia ${ }^{1}$, Lei Zhang ${ }^{1, a}$, Feng $\mathrm{Li}^{2}$, Dongying Zhang ${ }^{1}$, Delu Xu ${ }^{1}$, Zhiyong Wang ${ }^{3}$, Jianwei Chang ${ }^{1}$ and \\ Yusuo $\operatorname{Ren}^{1}$ \\ ${ }^{1}$ Shandong Electrical Engineering \& Equipment Group Co., Ltd (SDEE), 100071, Beijing, China \\ ${ }^{2}$ STATE GRID Corporation of China, 100031, Beijing, China \\ ${ }^{3}$ Department of Mechanics, Tianjin University, 300072, Tianjin, China
}

\begin{abstract}
A test system to measure the deformation of the transmission tower based on binocular vision method is introduced in this work. The test system can achieve the accurate monitoring of the transmission tower deformation, and provide deformation data for the reliability analysis of the transmission tower provides both in static and dynamic measurement. The test system is successfully applied in a tower type test.
\end{abstract}

\section{Introduction}

The deformation measurement and the estimate of load capacity for the towers in transmission lines are more and more important. In former years the deformation of transmission towers are usually studied using numerical method, because it can calculate the 3D deformation with low cost. However, researches shown that the numerical results cannot agree with the experiments very well under complex loading conditions[1,2]. Therefore, real scale tower test for transmission lines under several load cases are more and more performed to estimate the loading capacity in recent years. The measurement of the field deformations of transmission line towers in the real scale tower test is necessary.

The traditional deformation measurement technologies such as the displacement sensor and the strain gauge can only measure deformation in one-dimension within a very limited range at certain points which are selected before test, and it cannot get the full field of the transmission tower deformation $[2,3]$. Conventional sensors are usually contact-type which need to be installed on the structure to be tested that must bring certain damage to structure inevitably. In order to avoid these questions, optical measurement method has been reported by a lot of researchers [1]. In addition to its full-field and non-contact measurement capability, the 2D-DIC method (Digital Image Correlation) is well known for its ease of preparation,installation automatic processing, and pervasive applications. 2D-DIC method is mainly used to measure the in-plane displacements and strains. In order to overcome this limitation of DIC in two dimension measurement, researchers developed DIC into

${ }^{\text {a }}$ Corresponding author: zhanglei@sdee.sgcc.com.cn 
three-dimension coordinate (3D-DIC), in which the binocular vision method is introduced into DIC mainly[4].

This work proposes to use measure the deformations of a transmission tower using 3D-DIC, which can measure the position parameters in global coordinate with feature points of the tower. By using the calibration data of binocular cameras and image parallax maps, the $3 \mathrm{D}$ coordinates of any points and variations after deformation can be obtained.

\section{3D digital image correlation method}

\subsection{Binocular visions}

Fig. 1 (b) illustrates the main principle of binocular vision. $\mathrm{O}_{\mathrm{cl}}$ and $\mathrm{O}_{\mathrm{cr}}$ are the left and right optical centers of the two cameras respectively, see In Fig. 1 (b). An object point A is captured as the point $A_{1}$ and $A_{r}$ in the image planes of the two cameras respectively. The 3D-DIC is used to reconstitute the 3 dimensional coordinate values at point $A$ in global coordinate with respect to coordinate values of $A_{1}$ and $A_{r}$ in the coordinates of left and right cameras respectively. As shown in Fig. 1 (b), it is can be seen that researchers can not determine the $3 \mathrm{D}$ coordinates of point $\mathrm{A}$ from just one camera. Thus, in 3D-DIC, two cameras are generally used to capture the same points synchronously on the object to be tested from two positions in different directions.
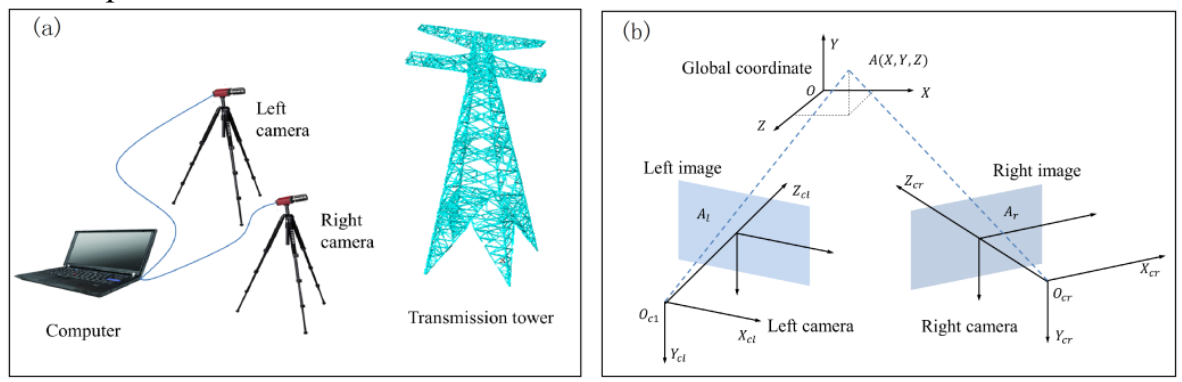

Figure 1. Schematic diagram of the 3D DIC system for measuring the deformation of towers

To measure the 3 dimensional values of the object point A which is a structure node on the tested tower, step 1 of the measurement is to establish a global coordinate. This step can be performed through the calibration of cameras. Step 2 is to calculate the coordinate disparity values of the same structure node of the tested tower between the left and right capures. With respect to the calibrated data of the left and right cameras and the measured coordinate disparity values between the two capure points $A_{1}$ and $A_{r}$, the $3 D$ coordinate values of the object point $A$ will be determined.

\subsection{D matching based on DIC}

The 3D matching is used to match the object node on the tower precisely in the left and right images imaged by the two cameras. The matching procedure is commonly considered as one of the most important parts in stereo vision method, can be performed using the subset-based matching technology which is adopted in the 2D-DIC method $[5,6]$. The principal concept of the 2D-DIC is to match the same object points with subsets between two images before and after load. In 2D-DIC, only one camera is used, and the position of the camera is fixed during measurement process. It is similar to the 2D-DIC method but with slightly differences. When performing the 3D-DIC, the 3D matching aims to match four subsets on the same object nodes captured by the two cameras with an angle between them before and after deformation of the object. In this work, structure nodes of transmission tower are selected as subsets to determine the displacements after deformation, see Fig. 2. 
In the subset-based matching method, the matching can be performed using matching intensity by minimizing or maximizing a function that includes the gray levels of pixels in the subsets in the images. The matching intensity value of a subset are correlated to one another until the intensity value reaches the $\mathrm{min} / \mathrm{max}$ value. The displacement of the object point, which represents the best possible match, can be determined once the $\mathrm{min} / \mathrm{max}$ value is obtained. In this work, the Zero-Normalized Sum of Squared Difference, see Eq. (1), was selected as the matching intensity function[7].

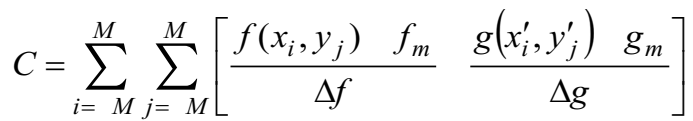

where,

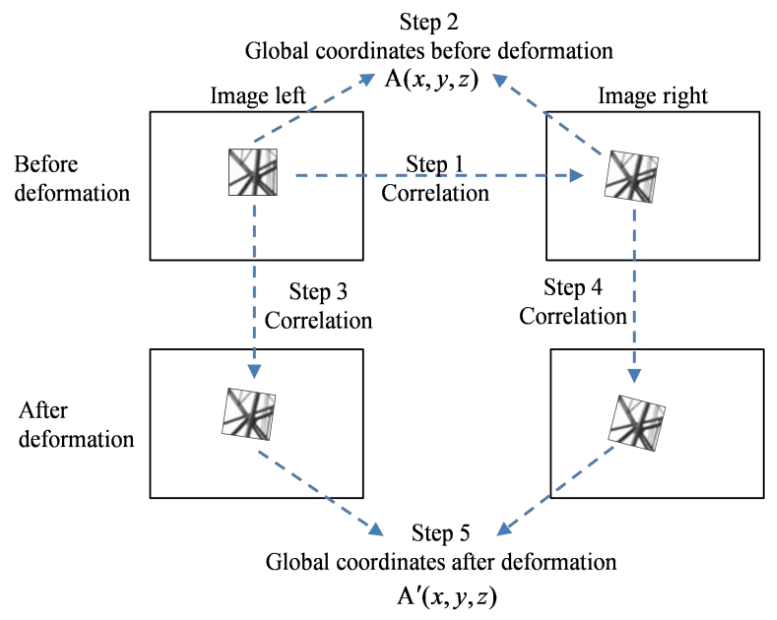

Figure 2. 3D-DIC method for matching a set of images captured by two cameras before and after load.

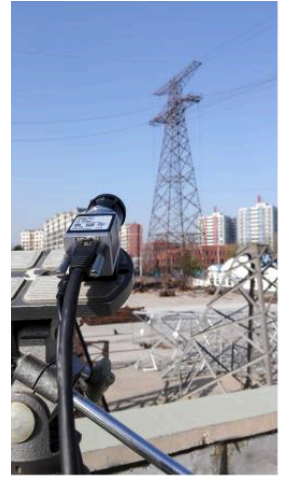

(a) Left camera

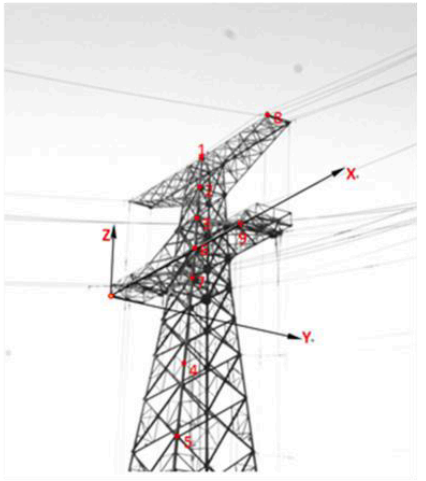

(b) Monitoring points of displacements

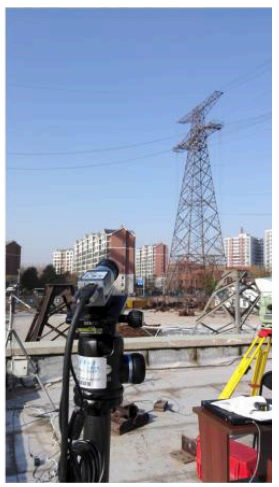

(c) Right camera

Figure 3. Monitoring points, left and right cameras in the real scale model test

Fig. 2 shows the steps used to measure the deformation using 3D-DIC. Once the binocular vision system is calibrated, the space positions and orientations of the two cameras are confirmed and the 3 dimensional positions of the object tower nodes in global coordinate can be calculated by multi-image triangulation.

Using the calibrated data of the two cameras fo the binocular vision system, the triangulation method has been used to calculate the 3 dimensional coordinate values of all the points matched by 3D-DIC. It is important that, prior to the application of triangulation process, all distortions at the 
object points in images should be corrected using the intrinsic parameters of the two cameras determined in the calibration process. Therefore, a more accurate 3D measurement can be obtained.

\subsection{Camera calibration}

Calibration of cameras is an important step in the 3D-DIC process, since there is a high dependence of the measurement accuracy on the parameters from camera calibration. The intrinsic parameters of cameras (principle point, effective focal length and lens distortion coefficient) can be determined in the camera calibration procedure. Moreover, in 3D-DIC, determination of the relative location and intersection angle between the left and right cameras are must be performed. In this work, the stereo vision method calibration technique is used followed by a nonlinear least-squares algorithm to optimize the calibration values of the camera parameters[8].

\section{Experiments}

The 3D-DIC system consists of 3 parts: two digital single-lens cameras with lens ( Basler acA1600 $20 \mathrm{gm}$ ), computer to calculate, shown in Fig. 1 (a). The size of CCD is $7.16 \mathrm{~mm} \times 5.44 \mathrm{~mm}$, the pixel size is $4.5 \times 4.5 \mu \mathrm{m}^{2}$, the resolution is $1600 \times 1200$ pixels, and the optical lens is COMPUTER lens of $16 \mathrm{~mm}$ focus.

The binocular vision system is applied in the real scale tower test of a transmission tower. The testing transmission tower is structured to be T-shaped for a single loop $\pm 500 \mathrm{kV} \mathrm{Dc}$ transmission line, see Fig. 3. The design parameters are as follows: practical height $48 \mathrm{~m}$, horizontal span $500 \mathrm{~m}$, vertical span $700 / 300 \mathrm{~m}$, ruling span $300 / 650 \mathrm{~m}$, maximum wind speed $27 \mathrm{~m} / \mathrm{s}$ and the maximum icing thickness $10 \mathrm{~mm}$. The type test was performed considering two typical working conditions. In the experiment, loads are imposed to the tower step by step on the basis of the designed working conditions. A total station is adopted to measure the deformation of feature points of the tower, which is used as a reference test. The monitoring points of displacement, which are selected at 9 structure nodes, are illustrated in Fig. 3 (b).

Table 1. Calibrated intrinsic parameters of cameras.

\begin{tabular}{|c|c|c|c|c|}
\hline & $\mathbf{f}_{\mathbf{x}}$ ( pixel ) & $\mathbf{u}_{\mathbf{0}}$ ( pixel ) & $\mathbf{f}_{\mathbf{y}}$ ( pixel ) & $\mathbf{u}_{\mathbf{0}}$ ( pixel ) \\
\hline Left camera & 3634.89 & 829.4 & 3635.76 & 608.11 \\
\hline Right camera & 3607.23 & 791.97 & 3609.96 & 633.86 \\
\hline
\end{tabular}

Table 2. Positions and orientations of left and right cameras in global coordinates.

\begin{tabular}{|c|c|c|c|c|c|c|}
\hline Left & $\mathbf{c}_{\mathbf{x}}$ & $\mathbf{c}_{\mathbf{y}}$ & $\mathbf{c}_{\mathbf{z}}$ & $\boldsymbol{\theta}$ & $\boldsymbol{\theta}$ & $\boldsymbol{\varphi}$ \\
\hline camera & $-6.7 \mathrm{~m}$ & $-1.7 \mathrm{~m}$ & $110.7 \mathrm{~m}$ & $53.2^{\circ}$ & $24.3^{\circ}$ & $11.0^{\circ}$ \\
\hline Right \\
camera
\end{tabular}

Table 3. displacements of the monitoring points under working condition 1. 


\begin{tabular}{|c|c|c|c|c|c|c|c|c|c|}
\hline $\begin{array}{c}\text { Monitoring } \\
\text { point }\end{array}$ & $\mathbf{1}$ & $\mathbf{2}$ & $\mathbf{3}$ & $\mathbf{4}$ & $\mathbf{5}$ & $\mathbf{6}$ & $\mathbf{7}$ & $\mathbf{8}$ & $\mathbf{9}$ \\
\hline $\mathbf{U}_{\mathbf{x}}(\mathbf{m m})$ & 152 & 115 & 107 & 89 & 25 & 107 & 112 & 144 & 100 \\
\hline $\mathbf{Y}_{\mathbf{y}}(\mathbf{m m})$ & 245 & 234 & 210 & 127 & 58 & 183 & 168 & 248 & 179 \\
\hline $\mathbf{U}_{\mathbf{z}}(\mathbf{m m})$ & -17 & -22 & -26 & -26 & -25 & -27 & -28 & -53 & -75 \\
\hline
\end{tabular}

Table 4. displacements of the monitoring points under working condition 2.

\begin{tabular}{|c|c|c|c|c|c|c|c|c|c|}
\hline $\begin{array}{c}\text { Monitoring } \\
\text { point }\end{array}$ & $\mathbf{1}$ & $\mathbf{2}$ & $\mathbf{3}$ & $\mathbf{4}$ & $\mathbf{5}$ & $\mathbf{6}$ & $\mathbf{7}$ & $\mathbf{8}$ & $\mathbf{9}$ \\
\hline $\mathbf{U}_{\mathbf{x}}(\mathbf{m m})$ & 410 & 364 & 343 & 196 & 118 & 305 & 284 & 418 & 312 \\
\hline $\mathbf{Y}_{\mathbf{y}}(\mathbf{m m})$ & 32 & 31 & 30 & 11 & 8 & 14 & 18 & 24 & 17 \\
\hline $\mathbf{U}_{\mathbf{z}}(\mathbf{m m})$ & -23 & -37 & -13 & -27 & -28 & -9 & -29 & -153 & -162 \\
\hline
\end{tabular}

\section{Results and discussion}

Using this camera calibration method, the intrinsic parameters of the cameras are determined, see Table 1. $\left(\mathrm{u}_{0}, \mathrm{v}_{0}\right)$ are the coordinates of the principle points of the cameras respectively; as well as $f_{x}$ and $f_{y}$ are the effective focal lengths of each camera along $X$ and $Y$ axes respectively. The intersection angle and relative location of the two cameras can also be obtained using their extrinsic parameters (Table 2).
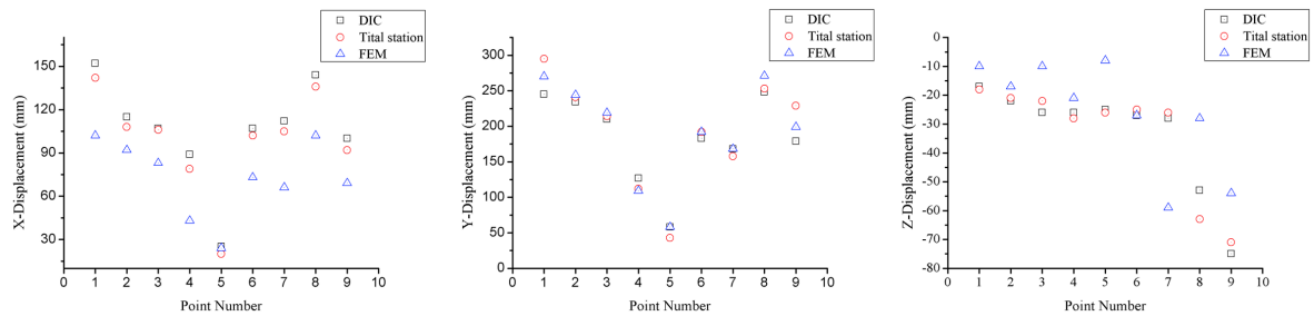

(a) Working condition 1
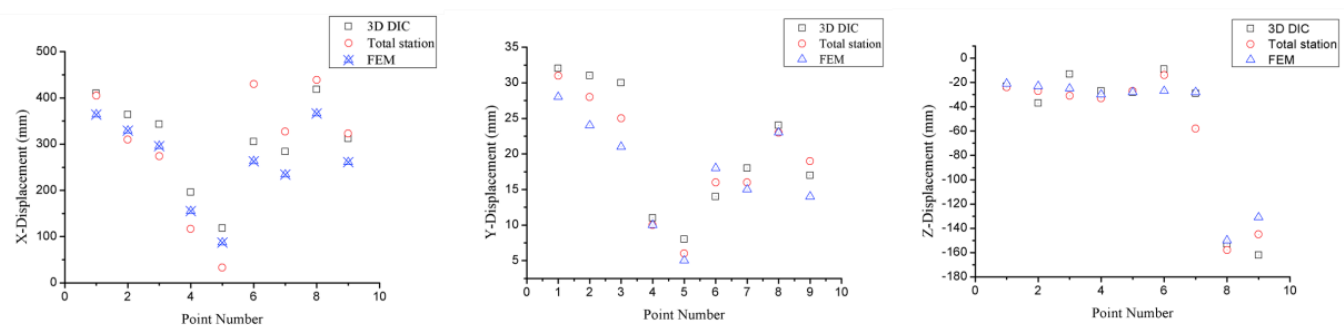

(b) Working condition 2

Figure 4. Comparison of the results from 3D DIC, total station and FEM. 
In order to obtain comparable measurement results with the total station, the same monitoring points (see Fig. 3 (b)) on the structure nodes were selected as the points for calculation. To ensure the accuracy of the 3D matching, all pixels within the reference subset, which is located at the current point calculated, should be large enough to cover the image characters around the current structure node. During the procedure of the correlation, the size of the subset was selected to be $31 \times 31$ pixels. Using the calibrated parameters in Tables 1 and 2, the monitoring node positions of the tower can be determined. The results are shown in Table 3 and Table 4.

To ensure the accuracy of measurement results, the results were compared with that obtained by a commercial 3D coordinate measurement machine (total station). The deformations are also calculated using finite element method (FEM) according to the tower's design report as anther reference. Fig. 4 (a) is the comparison of the displacement results from 3D DIC, total station under working condition 1. Fig. 4 (b) shows results under working condition 2 . The measurement results indicate that the displacements of the real scale tower measured using both of 3D DIC and total station are acceptable.

\section{Conclusions}

In this paper, a 3D DIC measurement system is introduced to measure the deformation of a transmission tower. The results of deformation obtained by the 3D DIC are compared with the results by both of the total station measuring and the finite element method calculation. This work shows the feasibility and effectiveness of the method, thus good prospect can be expected with it. As the trend of smart grid development, development of the intelligent detection technology for transmission line is necessary. As a commonly used technology in the visual field of artificial intelligence, the binocular vision technology proposed a new testing method for monitoring the running state of transmission towers quantitatively.

\section{References}

1. Z. Xiao, J. Liang, D. Yu, and J. Liu, Opt. Laser Eng. 48, 869 (2010).

2. B.-W. Moon, J.-H. Park, S.-K. Lee, J. Kim, T. Kim, and K.-W. Min, J. Constr. Steel Res. 65, 1 (2009).

3. J. Davis, D. Nehab, R. Ramamoorthi, and S. Rusinkiewicz, IEEE T. Pattern Anal. 27, 296 (2005).

4. K. Genovese, L. Casaletto, J. A. Rayas, V. Flores, and A. Martinez, Opt. Laser Eng. 51, 278 (2013).

5. B. Pan, H.-m. Xie, B.-q. Xu, and F.-1. Dai, Meas. Sci. Technol. 17, 1615 (2006).

6. Z.-F. Zhang, Y.-L. Kang, H.-W. Wang, Q.-H. Qin, Y. Qiu, and X.-Q. Li, Measurement 39, 710 (2006).

7. H. Shi, H. Ji, G. Yang, and X. He, Opt. Laser Eng. 51, 47 (2013).

8. T. Xue, B. Wu, J. G. Zhu, and S. H. Ye, Sensor. Actuat. A-Phys. 135, 185 (2007). 